\title{
Significación teológica y eclesial de Medellín y Puebla. Desafíos a los cristianos del primer mundo en el siglo XXI
}

\section{Juan José Tamayo-Acosta, Asociación de Teólogos Juan XXIII, Madrid.}

\section{Medellín y Puebla: entrada en el siglo XXI}

Han pasado treinta y un años de la II Conferencia General del Episcopado Latinoamericano, celebrada en Medellín (Colombia), y veinte de la III Conferencia, celebrada en 1979. Algunos pensarán que se trata de dos acontecimientos perdidos en la noche de los tiempos, que nada tienen que aportar o decir hoy, y menos aún de cara al futuro. Y lo mejor que puede hacerse es dejarlos en manos de los historiadores para que los incorporen en sus historias de la Iglesia latinoamericana como momentos importantes de su itinerario, pero nada más. Los cristianos y cristianas, teólogos y teólogas, saccrdotes, religiosos, religiosas y obispos han de olvidarse de ellos y mirar hacia adelante, intentando responder a los nuevos desafíos que nos depara el cambio de época que estamos viviendo. Así piensan algunos sectores cristianos posmodernos.

Habrá quienes crean que Medellín y Puebla fueron una pesadilla que ha durado demasiado tiempo y de la que ya es hora de librarse, o de una página incómoda que hay que anrancar o pasar de prisa, para volver a la Iglesia de la cristianidad, donde todo estaba en su sitio: los religiosos y las religiosas practicaban la obediencia; los teólogos seguían las orientaciones del magisterio eclesiástico; los cristianos acataban sumisamente las directrices de sus pastores hasta en sus más mínimos detalles; la Iglesia se dedicaba a cultivar su parcela religiosa, sin injerirse en la vida política y social, y menos aún en la economía, como hace ahora sin ton ni son, etc. Así pensarán algunos sectores cristianos - cada vez menos-, que asocian la identidad cristiana con el modelo de la cristianidad. 
No faltarán quienes sigan recordando y citando a Medellín y Puebla con profusión, pero haciendo de ellos una interpretación preconciliar y antiliberacionista y controlando su aplicación para evitar - dicen- que se vaya más allá de donde quisieron llegar ambas conferencias. Es una posición netamente eclesiástica, que consiste en citar los documentos aperturistas del magisterio papal y episcopal, pero dándoles hábilmente la vuelta y haciéndolos decir justamente lo contrario de lo que dicen. Así opera un importante sector conservador dentro de la Iglesia hoy.

Otros reconocerán la importancia y significación especiales de las dos con「erencias, pero limitándolas a América Latina. Su opinión es que poco o nada pueden aportar a otros continentes, donde la problemática y los desafíos son diferentes. En el fondo, se trata de una postura autosuficiente que no admite lecciones de nadie, porque cree que las suyas son las mejores. $Y$, en el caso de América Latina, con más motivo, ya que — se dice- fue un continente "descubierto" ( $i$ ?) y "evangelizado" por nosotros ( $i$ ?). Además, es pobre en todos los aspectos y poco puede enseñarnos en ninguna materia. Vuelve a repetirse la reacción de los judíos ante Jesús: “¿de Nazarel puede salir algo bueno?”.

Mi punto de vista difiere sustancialmente de las posiciones que acabo de describir. Creo que Medellín y Puebla son dos de los acontecimientos más relevantes de la Iglesia latinoamericana en toda su historia, que han tenido importantes repercusiones en todos los ámbilos de la vida de este continente, más allá de su dimensión religiosa: el social, el político, el económico, el cultural, el étnico y en otros entornos religiosos, culturales y políticos, más allá de América Latina. En otras palabras, cambiaron el rumbo de la Iglesia latinoamericana, que venía de una larga etapa de cristianidad, orientándola hacia la liberación y la inculturación. La liberaron de la vieja hipoteca colonial y le devolvieron la faz profética de los grandes evangelizadores.

Medcllín y Puebla constituyen un punto de referencia obligado para los países latinoamericanos, porque defendieron sin ambages los derechos humanos al liempo que denuncian sus violaciones; propician la democratización del continente, al tiempo que critican las tendencias populista y dictatoriales; y abogan por un modelo de desarrollo solidario al tiempo que critican al capitalismo; devuelven a esos países a sus propias raíces culturales, al tiempo que los orientan hacia la nueva civilización que entonces estaba gestándose ya.

El mensaje de Medellín y Puebla no sólo no está superado, sino que, en muchos aspectos, se ha quedado en el papel y no ha sido llevado a la práctica. Peor aún, desde el mismo Vaticano - la Congregación de Religiosos, la Congregación de Obispos, la Congregación para la Doctrina de la Fe, elc., y con el apoyo de influyentes sectores de la jerarquía eclesiástica y de la teología latinoamericanas - no se ha cesado de obstaculizar el proyecto liberador surgido de las Digitalizado por Biblioteca "P. Florentino ldoate, S.J."

Universidad Centroamericana José Simeón Cañas 
dos conlerencias y de denunciar a quienes, lieles al magisterio episcopal latinoaınericano y al Vaticano II, han intcntado convertirlas en criterio de su reflexión teológica y de su actuación pastoral.

Creo que los documentos de Medellín y Puebla siguen siendo, lodavía hoy -y lo serán más en el futuro-, un buen piograma para la renovación de las instituciones eclesiales y una buena guía para la regeneración de la vida política y la actividad económica. Conservan, por lo tanto. la misma actualidad o mayor que cuando se celebraron. Eso sí, deben ser leídos, interpretados y actualizados mirando al futuro y atendiendo a los cambios producidos en el mundo y en las propias sociedades del conlinentc.

Diría más, las dos conferencias anticiparon en veinte y treinta años la entráda de la Iglesia latinoamericana en el siglo XXI. De no haber sido por ellas, esa Iglesia se hubiera quedado no ya en el siglo $\mathrm{XX}$ - donde cultural y religiosamente apenas estuvo-, sino en el XIX.

No puedo ocuparme de lodos los aspectos positivos de ambas conferencias. Me centraré solamente en dos: las comunidades eclesiales de base y la reflexión teológica.

2. Las comunidades eclesiales de base, "focos de evangelización y motores de liberación"

Medellín y Puebla han tenido una importancia fundamental en el desarrollo y la evolución de las comunidades eclesiales de base. Mientras éstas eran consideradas un movimiento marginal y subterráneo en Europa y Estados Unidos, e incluso eran perseguidas por sus jerarquías, las dos conferencias latinoamericanas reconocieron su plena eclesialidad y las convirtieron en el quicio de la acción pastoral.

Las comunidades de base constituían ya un fenómeno sociológico, teológico y pastoral ampliamente difundido, con una presencia significativa en la Iglesia y la sociedad latinoamericanas. Algunos obispos las habían promovido, animado e incluso privilegiado como motor de renovación eclesial y cauce prioritario de evangelización liberadora. Veamos un ejemplo. En el encuentro de pastoral de la diócesis brasileña de Crateús, celebrando en 1967, siendo obispo monseñor Antonio Fragoso, se aprobaba la siguiente propuesta: "La comunidad de base es una respuesta a las exigencias de renovación de la persona. La educación y la responsabilidad sólo son posibles en grupos pequeños". Partiendo de este principio, se proponian estas tres prioridades pastorales: la Iglesia se articula en torno a las comunidades de base. la educación en la fe tiene lugar en el seno de esas comunidades, y la vida de las comunidades comporta la creación de ministerios eclesiales que han de ser ejercidos por los cristianos de cada comunidad.

Digitalizado por Biblioteca "P. Florentino Idoate, S.J."

Universidad Centroamericana José Simeón Cañas 
Pero el acto magisterial más explícito y de mayor autoridad pastoral en el reconocimienlo de las comunidades eclesiales de base tuvo lugar en la conferencia de Medellín. donde se considera a la comunidad cristiana de hase como "el primero y fundamental núcleo eclesial". Las comunidades eclesiales de base son presentadas como el vehículo de la vivencia de la comunión de los cristianos: "La vivencia de la comunión a que ha sido llamado el cristiano, debe encontrarla en su comunidad de base" (Documento de Medellin, "Pastoral de conjunto", N. 10). Constituyen "el punto clave en la pastoral de los misioneros que implantan la le y la Iglesia en nuestro continente" (ibid., N. 12). Por comunidad de base entiende Medellín "una comunidad local o ambiental, que corresponda a la realidad de un grupo homogéneo, y que tenga una dimensión tal que permita el trato personal fraterno entre sus miembros" (ibid. N. 10).

Entre las tareas cclesiales y sociopolíticas a asumir por las comunidades eclesiales de base cita las siguientes: responsabilizarse de la riqueza y expansión de la fe y del culto; ser "célula inicial de estrucluración eclesial y foco de evangelización"; actuar como "factor primordial de promoción humana y desarrollo". Medellín resalta la función de los líderes y dirigentes de las comunidaJes, que pueden ser sacerdotes, diáconos. religiosos, religiosas o laicos y que han de asumir responsabilidades "en un clima de autonomía" (ibíd., 11, subrayado mío).

Afirma la necesidad de formar el mayor número de comunidades en las parroquias, especialmente rurales, o en zonas urbanas de marginación, con estas características: estar basadas en la palabra de Dios, realizarse en la celebración eucarística, en comunicación con el obispo y bajo su dependencia, tener sentido de pertenencia y conciencia de una misión común, participar activa y conscientemente en la vida litúrgica y en la convivencia comunitaria. El apostolado de los laicos tendrá mayor transparencia de signo y mayor densidad eclesial si está apoyado en comunidades de fe, a través de las cuales acontece la Iglesia "en el mundo, en la tarea humana y en la historia" (ibid, N.12).

A partir de Medellín, las comunidades eclesiales de base ocuparon un lugar relevante en la eclesiología latinoamericana de la liberación. Ellas han asumido una doble tarea: re-inventar, re-engendrar nuevamente la Iglesia como comunidad de comunidades desde la experiencia de los pobres e integrarse en los procesos de liberación.

Diez años después, Puebla continúa el magisterio de Medellín y constata "la multiplicación y maduración de las comunidades eclesiales de base, que se han convertido en focos de evangelización y en motores de liberación y desarrollo" (Documentos de Puebla, N. 56). Ve en ellas "una de las fuentes de nacimiento de ministerios laicales: presidentes de asambleas, recompensables de comunidades, catequesis, misioneros" (N $\mathbf{N}$ Digitalizado por Biblibteca "P. Florentino Idoate, S.J."

Universidad Centroamericana José Simeón Cañas 
Entrc las funciones que Puebla asigna a las comunidades eclesiales de base, he aquí las más importantes:

- constituyen un ambiente propicio para el surgimiento de nuevos servicios laicos;

- en ellas se difunden la catequesis familiar y la educación en la le de los adultos, en formas más adecuadas al pueblo sencillo;

- sc acentúa el compromiso con la familia, el trabajo, el barrio y la comunidad local;

- Ios cristianos pueden vivir una vida más evangélica en el seno del pueblo;

- interpelan las raíces egoístas y consumistas de la sociedad y ofrecen un valioso punto de partida en la construcción de la nueva sociedad;

- son expresión del amor preferente de la Iglesia por la gente sencilla;

- en ellas se expresa y purifica la religiosidad;

- posibilitan la participación en la acción eclesial y en el compromiso de transformación del mundo;

- deben constituir un ejemplo de convivencia donde se aúnen libertad y solidaridad, se viva una actitud diferente ante la riqueza, se ensayen lormas nuevas de organización y estrucluras más participalivas y, sobre todo, se articulc la radical comunión con Dios y las diferentes formas de comunión humana.

Los obispos latinoamericanos reunidos en Puebla asumieron el compromiso de "promover, orientar y acompañar a las comunidades eclesiales de base".

Pero el mensaje de Puebla no es tan lineal como el de Medellín en torno a las comunidades eclesiales de base. Está lleno de prevenciones y sospechas, de desconlianzas y miedos. En la misma definición que da de ellas se aprecia ya un importante recorte a Medellín. Esıo sc confirma sólo con citar la definición: "La comunidad eclesial de base, como comunidad, integra ramilias, adultos y jóvenes, en íntima relación interpersonal en la fe. Como eclesial, es comunidad de le, esperanza y caridad; celebra la palabra de Dios y se nutre con la eucaristía, culmen de todos los sacramentos: realiza la palabra de Dios en la vida a través de la solidaridad y cl compromiso con el mandamiento nuevo del Señor y hace presente la misión cclesial y la comunión visible con los legítimos pastores, a través del servicio de coordinadores aprobados. Es de base, por estar constituida por pocos miembros, en forma permanente y a manera de célula de la gran comunidad" (N. 489). No hay en la defínición una sola referencia al componen-

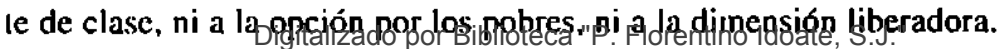


Tras conocer la falta de atención prestada al trabajo de formación de las comunidades eclesiales de base en algunos lugares, lamenta que "intereses visiblemente políticos pretendan manipularlas y apartarlas en algunos lugares de la auténtica comunión con los obispos" (N. 58). Cree, asimismo, que, al descuidar la formación de líderes educadores en la fe y de cristianos responsables en los organismos intermedios de barrio, del mundo obrero y campesino, hay miembros de comunidades o comunidades enteras radicalizadas ideológicamente que "van perdiendo el sentido auténlico eclesial" (N. 478).

Se hace una crítica severa de la "Iglesia popular", comenzando por considerar el nombre mismo poco afortunado, en el sentido de que aparece como distinla de "otra", la oficial o inslitucional. Ello implica, a juicio de los obispos reunidos en Puebla, introducir la división en el seno de la Iglesia y negar la función de la jerarquía" (N.162). Sin embargo, hacen un esfuerzo por salvar a la Iglesia popular si se entiende como "una Iglesia que busca encamarse en los medios populares del continente y... surge de la respuesta de fe que esos grupos dan al Señor" (N. 162).

La centralidad atribuida por Medellín a las comunidades eclesiales de base queda mermada en Puebla, que reconoce a la familia como "el primer centro de evangelización" (N. 467). Con todo, y a pesar del clima de sospecha de Puebla hacia las comunidades eclesiales de base, hay un reconocimiento explícito de las mismas, que permite hablar de una continuidad básica, aunque muy matizada, con Medellín.

\section{Protagonismo de los seglares y desclericalización de la Iglesia}

El reconocimiento de las comunidades eclesiales de base por parte de Medellín y Puebla ha tenido importantes consecuencias en el cambio de las estructuras de la Iglesia católica, en su organización interna y en su dinámica pastoral. Con la centralidad de la comunidades eclesiales de base, la Iglesia comienza a estructurarse en torno a los carismas, y no desde la jerarquía, que otrora constituía su principio rector y vertebrador. Estamos ante una aplicación coherente y decidida del Capítulo Il sobre "El pueblo de Dios", de la constitución Luz de las gentes, del concilio Vaticano II. La Iglesia deja de girar en torno a los ministerios clericales, sobre los que se sustentaba la organización eclesial, y lo hace a partir de los ministerios laicales. Los seglares asumen el protagonismo dentro de las comunidades y ejercen responsabilidades directivas, proféticas y educativas, difícilmente pensables antes de las dos conferencias. Tal protagonismo no debe entenderse como concesión graciosa de la jerarquía al pueblo o del clero a los laicos. Emana de la igualdad de todos los cristianos y cristianas por el bautismo, de la común dignidad de hijos de Dios, de la pertenencia a la misma comunidad de fe y de la corresponsabilidad como miembros activos del pueblodtyetdDieslo por Biblioteca "P. Florentino Idoate, S.J." 
Los dos capítulos cilados quc, al decir del cardenal Suenens, supusieron una "revolución copernicana" en la eclesiología, apenas tenían cauces de viabilidad, pues se veían obstruidos por el Capítulo III sobre "La jerarqula", de la misma constitución, que reproducía el modelo jerárquico piramidal de antes del concilio.

La eclesiología de comunión en torno a las comunidades eclesiales de base, de Medellín y Puebla, lograba desbloquear la situación eclesiológica creada por el Vaticano II y evitaba las rupluras y los desencuentros producidos en las iglesias europeas entre la jerarquía y los movitnientos cristianos de base. En América Latina no hay lugar ni para la ruptura ni para la yuxtaposición de dos modelos de Iglesia, el comunitario y jerárquico. Jerarquía y comunidad eclesial de base no se presentan como dos realidades enfrentadas u opuestas. Son, más bien, dos dimensiones de la única Iglesia, que se lecundan y enriquecen muluamente, dos funciones eclesiales que convergen en el horizonte del reino de Dios y en el servicio a los pobres y marginados.

La experiencia de las comunidades eclesiales de base en América Latina constituye un ejercicio práctico de superación de la oposición clérigos-laicos y de desarrollo del binomio comunidad-ministerios.

\section{Impulso a la teología de la liberación}

Medellín y Puebla no fueron conferencias teológicas en el sentido estricto del término. Tampoco pretendieron serlo. Tanto las ponencias como la orientación general tuvieron carácter sociológico y pastoral. Aun así y todo, hubo una presencia importante de teólogos que asesoraron a los obispos y dejaron su impronta en los textos. Muchos de los obispos ponentes de Medellín destacaron posteriormente en el compromiso por llevar a la práctica las grandes líneas de la conferencia en el día a día de su trabajo pastoral, que intentó compaginar armónicamente evangelización y liberación, y que dio lugar a uno de los programas de evangelización liberadora más esperanzadores para toda América Latina, y en el apoyo explícito a la teología de la liberación, en los momentos en que más arreciaba la persecución contra ella, tanto desde el Vaticano y desde las lilas de la jerarquía latinoamericana como desde los poderes económicos, políticos y militares. Entre los ponentes de Medellín cabe citar a Marcos G. McGrath, entonces obispo de Santiago de Veraguas (Panamá) y posteriormente arzobispo de Panamá: Eduardo E. Pironio, entonces obispo auxiliar de Buenos Aires (Argentina) y secretario general del CELAM y posteriormente presidente del CELAM; Samuel Ruíz, obispo de Chiapas (México); Leónidas E. Proaño, obispo de Riobamba (Ecuador).

El método inductivo seguido en Medellín inspiró la metodología de la teología de la liheración, entonces en ciemes. La conferencia comenzó con una "vi-

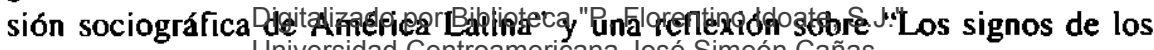
Universidad Centroamericana José Simeón Cañas 
tiempos en América Latina". Siguió con una interpretación cristiana de dichos signos, que consideró el hoy de América Latina no como un estigma, sino como un tiempo privilegiado de salvación. Luego, trató sobre la relación entre Iglesia y promoción humana. Abordo, finalmente, algunos aspectos concretos, como la evangelización, la pastoral de masas y la de élites, la unidad y las tensiones en la Iglesia y la coordinación pastoral.

Con Medellín y Puebla, la teología supera la larga etapa colonial -durante la que fue simple remedo de la neoescolástica decadente- la más efímera etapa desarrollista, y entra en la órbita de la liberación como respuesta a la principal urgencia del continente: la transformación de las estructuras injustas generadoras de pobreza y opresión entre las mayorías populares. Y ello no como un cambio oportunista, sino en respuesta al "sordo clamor [que] brota de millones de hombres [y mujeres, habría que añadir], pidiendo a sus pastores una liberación que no les llega de ninguna parte" y a "las quejas de que la jerarquía, el clero, los religiosos, son ricos y aliados de los ricos" (Medellin, "La pobreza de la Iglesia", N. 2). Puebla volverá a la idea del "grilo" y dirá que "es ahora más claro, creciente, impetuoso y, en ocasiones, amenazante" (N. 88).

Si el Vaticano II invitaba a los creyentes a estar presentes en el mundo como levadura en la masa y a hacer creíble la fe entre los no creyentes, Medellín y Puebla llaman a los cristianos y cristianas del continente latinoamericano a estar presentes en el mundo de los pobres y a hacer creible la fe, optando por los pobres, a través de la presencia en los movimientos de liberación.

La impronta de los pobres quedará grabada a fuego en la teología latinoamericana de los décadas siguientes, que logrará credibilidad no sólo en el propio continente, sino en otros ámbitos, donde la situación estructural de la pobreza no es tan sangrante, pero sí lo suficientemente preocupante como para interpelar la conciencia de los cristianos proféticos y para invitar a los teólogos y las teólogas a reformular la fe cristiana, en clave de liberación.

Con Medellín, la teología latinoamericana recuperó amplios espacios de libertad, al menos por un tiempo y dentro del propio continente, y se abrió al pensamiento crítico, en la línea propuesta por el Vaticano II. "El espíritu crítico más agudizado la purifica [a la vida religiosa] de un concepto mágico del mundo y de residuos supersticiosos, exigiendo cada vez más una adhesión verdaderamente personal y operante de la fe (Constitución sobre la Iglesia en el mundo actual, N. 7). Ser teólogo o teóloga no es corear las consignas del magisterio eclesiástico, ni comentar críticamente las encíclicas papales, sino repensar crf́licamente la fe para dar razón de la misma, en cada contexto histórico. Desgraciadamente, cuando las teólogas y los teólogos latinoamericanos comenzaron a ejercer su función intelectual de una manera crítica, la Sagrada Congregación para la Doctrina de la Fe inició una elapa de controles, sospechas, proce-

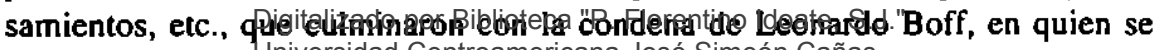


condenaba a cuantos tcólogos y tcólogas transitaban por las amplias veredas abiertas por el concilio Vaticano II, Medellín y, en menor medida, Puebla.

Gracias al impulso de Medellín, la teología en América Latina dejó de ser simple mímesis de la teología europea y norteamericana -conservadora o progresista-, adquirió identidad propia y supo responder con rigor cientílico y fucrza profética a los succsivos desafíos de la realidad latinoamericana: desde la experiencia de pobreza de las mayorías populares; desde la perspectiva de la mujer doble o iriplemente oprimida; desde el mundo de los negros e indígenas, cuya cultura es negada sistemáticamente; desde la inserción y el compromiso con cl campesinado; desde la solidaridad con los niños de la calle; en diálogo crítico con la cconomía, la cultura y la política, ctc. Es, en fin, una teología inculturada en esos mundos a partir de los nuevos sujelos colectivos de la le.

Los Ireinta años posteriores a Medellín han sido los más fecundos y creativos, teológicamente hablando, de la historia de la Iglesia latinoamericana. Medellín contribuyó a cambiar el modo de hacer tcología. El nuevo mélodo seguido es el induclivo parte de la experiencia y no de enunciados docirinales. Su palabra primera es la realidad, analizada con rigor crítico desde las ciencias sociales y humanas. La realidad es interpretada a la luz de la $\mathrm{re}$, intentando descubrir tanto los signos liheradores del reino de Dios como los antisignos, que se oponen a la realización del reino de Dios en la historia. El tercer momento es la praxis, que era también el momento primero, junto con la contemplación.

Medellín y Puebla han ayudado a descubrir la importancia del lugar social de toda teología, cuestión descuidada por los propios teólogos por considerarla irrelevante. No todos los lugares son igualmente válidos para hacer teología. Hay uno privilegiado, que coincide con el de la revelación de Dios y el de Jesús de Nazaret: los pobres. La reflexión tcológica no parte de cero, ni se hace desde las nubes. Responde siempre a unos intereses. Parte de una determinada precomprensión y presupone unas determinadas opciones. No es, por tanto, neuIral ni ética, ni politica, ni social. Lo que hizo Medellín lue explicitar esas opciones y esos intereses, que se concretan en la opción por los pobres y definir los dos desafíos a los que había de responder la teología: la inculturación y la liberación.

Este método ha sido seguido posteriormente en otros continentes, como Asia y Africa, si bien de manera creativa y leniendo en cuenta la realidad propia de csos continentes, y ha mutado el modo de hacer teología en otros entornos culturales y sociales caracterizados por la marginación y la exclusión sociales. 


\section{Desafios a los cristianos y cristianas del primer mundo}

Las conferencias del episcopado latinoamericano celebradas en Medellín y Puebla no están ahí sólo para ser recordadas y admiradas -aunque bueno es recordarlas y admirarlas-, sino para ser seguidas en sus líncas fundamentales, si hien no miméticamente. sino de forma creativa en los nuevos contextos socioculturales y eclesiales. Reproducirlas escolarmente fuera del entorno cultural y religioso en que surgicron me parece un acto de pereza intelectual, amén de un anacronismo. Pero desconocerlas. me parece un aclo de olvido, que nos llcvaría, tarde o temprano, a repetir el cristianismo colonial de cristianidad. Y eso es mayor anacronismo lodavía. Medellín y Puebla forman parte de nuestra historia eclesial y como tales hemos de asurmirlos y heredarlos, pero no sólo en su contenido, sino en los relos que nos lanzan y a los que debemos responder en cl presente y en el futuro. He aquí los que considero más importantes para las iglesias, los cristianos y las cristianas y los teólogos y las téologas del primer mundo, nosotros.

\subsection{Del individuo a la comunidad}

Una de las características de nuestras sociedades desarrolladas es el individualismo, que, en su afán por alirmar ilimitadamente al individuo, ha terminado por negar el principio de la alteridad y el carácter comunitario de la persona. Lo fundamental para el individuo es declinar el pronombre personal de primera persona en todos los casos del singular.

Pues bien, frente a la reclusión de la persona en el individualismo, Medellín y Puebla muestran una gran sensibilidad hacia la dimensión comunitaria de la existencia humana y al carácter solidario que emana directamente de la fe cristiana y forma una unidad con ella. Su más viva expresión son las comunidades eclesiales de base, de las cuales me he ocupado extensamente en el segundo apartado de este artículo. A partir de la vivencia comunitaria, el cristianismo latinoamericano está contribuyendo elicazmente a reconstruir las alteridades negadas: culturas pisoteadas, mujeres discriminadas, clases expoliadas, razas sojuzgadas y religiones olvidadas.

\subsection{De la civilización de la riqueza a la cultura de la austeridad}

El neoliberalismo económico, que ha triunfado en las sociedades desarrolladas y se impone cada vez con más fuerza en América Latina, propicia una civilización de la riqueza sustentada en tres pilares: la acumulación privada del capital como base fundamental del crecimiento económico; la posesión individual como base fundamental de la seguridad y el consumo como base de la propia seguridad y autoafirmación.

Digitalizado por Biblioteca "P. Florentino Idoate, S.J."

Universidad Centroamericana José Simeón Cañas 
Medellín y Puebla cuestionan de raíz. con distintos acentos, la civilización de la riqueza y del neoliberalismo, porque es selectiva y discriminatoria $y$, en consecuencia, no es universalizable. Los teólogos hispano-salvadoreños Ignacio Ellacuría -mártir- y Jon Sobrino reformulan el mensaje de Medellín y Puebla y proponen como alternativa al neoliberalismo una "civilización de la austeridad compartida", cuyo principio rector de las relaciones humanas no puede ser la acumulación de capital y el disfrute individual de la riqueza, sino la satisfacción de las necesidades básicas de todos los seres humanos del planela y la solidaridad compartida.

El paso de una civilización de la riqueza a una cultura de la austeridad compartida conlleva, en nuestras sociedades desarrolladas, la puesta en marcha de lo que Metz llama una "revolución antropológica", que nos exige:

Liberamos de nuestra riqueza y bienestar sobreabundantes, de nuestro consumo, en el que finalmente nos consumimos, de nuestra prepolencia, de nuestro dominio, de nuestra apalía..., de nuestra inocencia o, mejor dicho, de aquel delirio de inocencia que ha expandido hace ya mucho la vida de dominio en nuestros espíritus.

\subsection{De la retórica de los derechos humanos a la defensa de los derechos de los pobres}

En el tercer mundo resulta especialmente llamativa y cada vez más creciente la contradicción entre las declaraciones formales de los derechos humanos y la negación real de los derechos de los pobres. La sólida fundamentación filosófica, jurídica y política de los derechos humanos y sociales no resulta fácilmente compatible con la transgresión permanente de dichos derechos entre las mayorías populares del tercer mundo y los sectores marginados del primer mundo.

Si no se corrige a tiempo esta dramática contradicción, puede suceder que el discurso humanista que sirve de base a las declaraciones de los derechos humanos se convierta en instrumento de legitimación de todo tipo de discriminaciones. Poniendo ante nuestros ojos la injusticia estructural y haciendo llegar hasta nuestros oídos el clamor de los pobres, Medellín y Puebla desenmascaran la retórica vacía en que vienen envueltas las referidas declaraciones e intentan llenarlas de contenido real, abogando por los derechos humanos y sociales de los pobres.

\subsection{Del "fuera de la Iglesia no hay salvación" al "fuera de los pobres no hay salvación"}

Durante siglos imperó el eclesiocentrismo. La Iglesia se consideraba la única

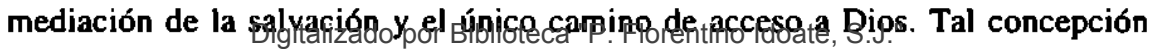


absoluta de la Iglesia quedó plasmada en la lómula de san Cipriano: "fuera de la Iglesia no hay salvación". Se tralaba de una visión exclusivista y excluyente, que tenía su analogado principal veterolestamentario en la imagen del "arca de Noé".

El criterio eclesiocéntrico exclusivista lue superado por el Vaticano II, que ofreció una visión inclusiva de la salvación, no mediada sólo por la pertenencia a la lglesia católica visible, sino abierta a otras mediaciones antropológicas, religiosas y cosmocéntricas. El nuevo enfoque más abierto se expresa en la lórmula de E. Schillebeeck: "fuera del nuundo no hay salvación".

El planteamiento de Medellín y Puebla sobre la mediación de la Iglesia y del mundo en la salvación lleva a relormular los principios precedentes, a partir de la opción por los pobres de esta guisa: "fuera de los pobres no hay salvación". Se pasa de la centralidad de la Iglesia o del mundo en abstracto a la centralidad de los marginados. Con cllo quiero significar no sólo que los pobres acceden de manera preferente a la salvación, sino que, en palabras de Ignacio Ellacuría, "son también los salvadores por antonomasia, los que van a realizar la verdadera salvación y la liberación integral".

\subsection{De la historia como progreso a la historia como cautividad}

La ilustración entiende la historia como un proceso progresivo hacia la emancipación humana, que culminará con la realización definitiva del ser humano ideal; pero no de todos los seres humanos, sino de los que han accedido a la cima de la racionalidad moderna. En esta concepción de la historia y de la razón no caben ni la razón de los vencidos ni la razón que brota del sufrimiento ajeno.

Medellín y Puebla corrigen dicha concepción optimista y entienden la historia no a partir de sus éxilos, sino de sus costes humanos, traducidos en miseria, pobreza, injusticias, muertes prematuras, enfermedades, analfabetismo, violencia estructural, etc. Son situaciones de marginación a las que Puebla pone rostros:

- rostros de indfgenas y con frecuencia también afroamericanos, que, viviendo marginados y en siluaciones inhumanas, pueden ser considerados los pobres entre los pobres;

- rosiros de campesinos. que como grupo social viven relegados en casi todo nuestro continente, careciendo de tierra, en situación de dependencia interna y externa, sometidos a sistemas de comercialización que los explotan;

- rostros de obreros con frecuencia mal retribuidos y con dificultades para organizarse y defender sus derechos;

- rostros de marginados y hacinados urbanos, con el doble impacto de la carencia de bienes materiales, frente a la ostentación de la riqueza de

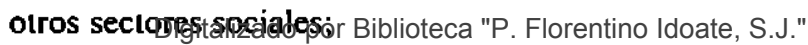

Universidad Centroamericana José Simeón Cañas 
- rostros de subempleados y desempleados, despedidos por las duras exigencias de crisis cconómicas y muchas veces de modelos de desarrollo que someten a los trabajadores y sus lámiliares a lirios cálculos económicos;

- rostros de jóvenes, desorientados por no encontrar su lugar en las sociedad y frustrados, sobre todo en zonas rurales y urbanas marginales, por lalla de oportunidades de capacitación y ocupación:

- rostros de niños, golpeados por la pobreza. desde antes de nacer, por trabárscles sus posibilidades de realizarse a causa de deliciencias mentales y corporales irreparabics que los acompañarán toda su vida; los niños vagos (de la calle) y muchas veces explotados de nuestras ciudades, fruto de la pobreza y la desorganización moral lamiliar;

- rostros de ancianos. cada día más numerosos, frecuentemente marginados de la sociedad del progreso, que prescinde de las personas que no producen (N. 20).

La historia avanza, es verdad, pero no al mismo ritmo para todos. El progreso selectivo y excluyente: es progreso de los menos y regreso de los más.

La historia, leída desde los pobres, es vista como cautiverio, y no como triunfo universal. De ahí que mostremos nuestro acuerdo con el siguiente texto de Walter Benjamín: "La Iradición de los oprimidos nos enseña que la regla de la historia es el estado de excepción en el que vivimos". Ahora bien, el grito de los pobres, de que habla Medellín, no es un grito resignado, sino esperanzado; es un clamor por la liberación. América Latina, constata Medellín, se encuentra "en el umbral de una nueva época histórica. llena de una anhelo de emancipación total, de liberación de toda servidumbre, de maduración personal y de integración colectiva" (Imtroducción, N. 4). En el continente se está gestando, dolorosamente, una nueva civilización, que Medellín interpreta como "un evidente signo del Espíritu" (ibid.) Y en la que ve "las huellas de la imagen de Dios en el hombre, como un potente dinamismo" (ibíd.).

\subsection{De la razón instrumental a la razón compasiva}

La teología es inteligencia de la fe y, como tal, debe trabajar con la cabeza, y cuanto más lúcidamente lo haga, mejor. No puede diluirse en testimonialismo. En un clima cultural secularizado se hace más necesario que nunca dar razón de la fe. Pero la razón de la fe no puede reducirse a una justificación racionalista de vía estrecha, que elimine del discurso teológico el universo simbólico, la experiencia religiosa, la dimensión práctica de la fe y la razón del corazón.

Amén de inteligencia de la fe, la teología es inteligencia de la esperanza y de la caridad. En ese sentido, el quehacer teológico debe entenderse, vivirse y

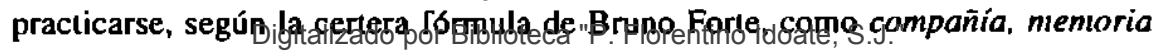


Y profecía. En olras palabras, la teología debe guiarse, además de por el principio esperanza (Bloch y Moltmann) y el principio aınor (san Juan, san Aguslín), por el principio misericordia o principio compasión (S. Weil, J. Sobrino).

\subsection{De una Iglesia culturalmente monocéntrica a un cristianismo culturalmente policéntrico}

J. B. Metz habla de la necesidad de pasar de una Iglesia monocéntrica, como la Iglesia curopea y norteamericana, a una Iglcsia culturalmente policéntrica. El parte del supuesto de que la Iglesia "es" Iglesia del lercer mundo con una prolohistoria europea occidental. El cristianismo europeo está tocando a su lin y se desplaza a pasos agigantados al tercer mundo. donde cuenta con el mayor número de creyentes en Cristo. Si hace un siglo cl 77 por ciento de los cristianos y cristianas estaba en el primer inundo y sólo el 23 por ciento vivía en cl tercer mundo, a las puertas del siglo XXI los porcentajes se han invertido: el 70 por ciento está en el tercer mundo y sólo el 30 por ciento se encuentra en el primer mundo.

Medellín contribuyó muy eficazmente a llevar a cabo el giro hacia un cristianismo culturalmente policéntrico, al encarnar la le cristiana en las señas de identidad del continente latinoamericano. Lo que surgió de aquella conferencia fue una Iglesia autónoma, liberada de la tutcla opresiva de las iglesias europeas, y muy especialmenle de la Iglesia española. Ahora bien, esa autonomía no significaba ruptura, sino realización especílica de la Iglesia, en un lugar concreto, con un dinamismo evangélico y un talante profético de mayor nivel que en las vicjas iglesias europeas. Así se manifestaba la universalidad del cristianismo sin caer en forma alguna de neocolonialismo o imperialisno. 